\title{
Anomalous Circulation Patterns in Association with Summertime Regional Daily Precipitation Extremes over Northeast China
}

\author{
Chuanlei Chen, ${ }^{1,2}$ Zhaoyong Guan $\mathbb{D}^{1},{ }^{1}$ Min Jiao, ${ }^{3}$ and Pengyu Hu $\mathbb{D}^{2}$ \\ ${ }^{1}$ Key Laboratory of Meteorological Disasters of Ministry of Education, \\ Collaborative Innovation Center for Meteorological Disaster Prediction, Early Warning and Assessment, \\ Joint Laboratory for International Cooperation on Climate and Environmental Change, \\ Nanjing University of Information Science and Technology, Nanjing 210044, China \\ ${ }^{2}$ Liaoning Meteorological Disaster Monitoring and Warning Center, Shenyang 110166, China \\ ${ }^{3}$ Liaoning Institute of Meteorological Sciences, Shenyang 110166, China
}

Correspondence should be addressed to Zhaoyong Guan; guanzy@nuist.edu.cn

Received 26 March 2019; Revised 18 July 2019; Accepted 26 August 2019; Published 28 October 2019

Academic Editor: Anthony R. Lupo

Copyright $(92019$ Chuanlei Chen et al. This is an open access article distributed under the Creative Commons Attribution License, which permits unrestricted use, distribution, and reproduction in any medium, provided the original work is properly cited.

Based on the NCEP/NCAR daily reanalysis product, NOAA monthly sea surface temperature (SST) dataset, and daily precipitation data collected at observational stations provided by Meteorological Information Center of China Meteorological Administration, the present study analyzes characteristic circulation anomalies conducive to regional extreme daily precipitation in the summer over Northeast China. Results indicate that 26 extreme precipitation events occurred during the 36-year period of 1979-2014. The precipitation threshold for the $99^{\text {th }}$ percentile is $22.2 \mathrm{~mm} / \mathrm{d}$, and the maximum extreme daily precipitation occurred in Liaoning Province. When extreme precipitation occurs, Northeast China is located to the southeast of an anomalous cyclonic circulation and northwest of an anomalous anticyclonic circulation, where a strong convergence zone is formed in the lower troposphere. The low-level convergence and upper-level divergence as well as the baroclinic circulation structure are favorable for the development of extreme daily precipitation. Meanwhile, there exist two symmetrical meridional circulations to the north and south of Northeast China. The two meridional circulations share the same ascending branch, which is conducive to the occurrence of precipitation. Located in the strong convergence zone to the southeast of the abnormal cyclonic circulation and northwest of the abnormal anticyclonic circulation, huge amounts of water vapor from the Inner Mongolia, Russia, the Japan Sea, and the mid-high latitudes of the Northwest Pacific are transported to Northeast China, providing sufficient water vapor condition for the occurrence of extreme daily precipitation in this region. In addition, daily extreme precipitation in Northeast China is also closely related to the accumulation and convergence of disturbance energy in Northeast China. It may also be related to the abnormal SST distribution that is high in the north and low in the south. Corresponding to extreme daily precipitation, SST anomaly in the Pacific Ocean is roughly characterized by the feature of "positive-north and negative- south" with the equator as the boundary.

\section{Introduction}

Extreme precipitation events occur frequently in recent years, and disasters like floods and mudslides induced by extreme precipitation cause serious impacts and damages on agricultural production, human life and economic development [1]. For this reason, great attention has been paid to extreme precipitation study worldwide. For Northeast China, summer is the season when precipitation is concentrated; it is also the main growing season for crops [2]. As the largest commercial grain production base in China, rainstorms and flood disasters caused by extreme precipitation in the summer have become one of the important factors affecting the grain production in Northeast China. 
Therefore, studies of formation mechanisms for extreme precipitation in Northeast China and its variation can not only deepen our scientific understanding of extreme precipitation, but also provide theoretical basis for forecasting summer extreme precipitation events in Northeast China. More important, such studies can provide useful references for government decisions about food production safety and disaster prevention and reduction.

Previous studies have revealed characteristic spatialtemporal variations of extreme precipitation in various regions of China [3-14]. These studies have shown that the long-term change trend of occurrence frequency of extreme precipitation is region-dependent $[6,12,14]$, while the occurrence frequency and intensity of annual extreme events $[13,15]$ and summer extreme events [7-11] are spatiotemporally inhomogeneous. Spatially, there exist large regional differences; temporally, significant interannual and interdecadal variations can be detected. In addition, changes in extreme precipitation have demonstrated obvious seasonal differences $[3,16]$.

Applying numerical models to study extreme precipitation is one of the main research approaches in recent years. Based on two observational datasets, 17 CMIP5 (Coupled Model Intercomparison Project Phase 5) global model simulations and nine RCM (regional climate model) outputs, Qin and Xie [17] studied the relationship of extreme precipitation in arid and humid regions of China with sea surface temperature (SST) in the equatorial eastern Pacific. Their results indicate that compared with observations the CMIP5 ensemble simulation can well reproduce the spatial and temporal patterns of extreme precipitation in both arid and humid regions of China, while RCMs can only simulate the temporal variation of extreme precipitation. In arid regions of China, all the datasets show a positive correlation between extreme precipitation index and SST in the equatorial eastern Pacific; in humid regions of China, however a more complicated relationship is found between extreme precipitation index and SST. Zhou et al. [18] compared and evaluated two objective prediction models for their simulations of the persistent rainfall event occurred in the Yangtze-Huaihe River Valley in the summer of 2016. Based on the CMIP5 global models, Li et al. [19] and Wu et al. [20] analyzed the characteristics of extreme precipitation index simulated by the models and evaluated the performance of these models on the simulation of extreme precipitation.

Circulation anomalies conducive to extreme precipitation in China are complicated. Chen and Zhai [21] investigated the structures and evolutions of the South Asia High in the upper troposphere, the blocking highpressure systems in the Ural Mountains and the Sea of Okhotsk in the middle troposphere and cyclonic circulation anomalies in the lower troposphere about 1-2 weeks prior to the occurrence of extreme precipitation in central and eastern China. Guan et al. [7] and Ke and Guan [10] respectively analyzed circulation anomalies conducive to extreme daily precipitation in the summer over the middle and lower reaches of Yangtze River and central China. Mechanism studies for the formation of extreme precipitation in China indicate that summer extreme precipitation in eastern China is closely related to atmospheric internal dynamics and energy propagation [14]. The intensity and location of the South Asia High exhibit significant impacts on extreme precipitation in eastern China $[21,22]$. Tropical cyclones also have important influences on extreme precipitation in China, and these influences are jointly modulated by ENSO and the western Pacific subtropical high (WPSH) $[23,24]$. In non-ENSO years, inland areas are more susceptible to extreme precipitation events caused by tropical cyclones, while in the El Niño years, tropical cyclones landing in China are fewer than normal, and the frequency of extreme precipitation events in China reduces correspondingly [23, 24]. Meanwhile, tropical cyclone tracks would shift southward when the WPSH extends westward, leading to more extreme precipitation events in South China [23, 24]. Bi et al. [25] found that more extreme precipitation events occur in the years when the super El Niño event ended. Sun et al. [26] pointed out that the joint effect of 10-30-day and 3060 -day intraseasonal oscillations (ISO) is one of the major reasons for the occurrence of the persistent rainfall in the Yangtze River valley in 1998.

As mentioned above, there are a lot of studies on both extreme precipitation in China and the relationships of precipitation in Northeast China with Northeast China cold vortex activities as well as ENSO (e.g., [27-29]). However, studies on regional extreme precipitation events in Northeast China are still very few. Particularly, as extreme precipitation events often occur over the period of June-September (e.g., [4]), the characteristics and mechanisms of variations of regional daily precipitation extremes (RDPE) in Northeast China in boreal summer still remain unclear. Therefore, in the present paper, we will explore the anomalous circulation patterns along with the possible formation mechanisms of RDPE events occurred in Northeast China during the recent decades.

\section{Data and Method}

2.1. Data. The data used in the present study include (1) the NCEP/NCAR reanalysis product of daily horizontal winds, vertical velocity, mixing ratio, and surface pressure, etc., for 1979-2014 [30]. The data have horizontal resolution of $2.5^{\circ} \times 2.5^{\circ}$; (2) monthly SST data from NOAA over the period 1979-2014 with horizontal resolution of $2^{\circ} \times 2^{\circ}$; and (3) daily precipitation data collected at 97 stations in Northeast China over the period of 1979-2014, which is provided by National Meteorological Information Center of China Meteorological Administration.

Summer refers to June-August, and the climatological mean refers to the 30-year averaged value over 1981-2010. Anomaly means the deviation from the climatological mean.

Northeast China is defined as the large area in China that is located to the east of $118^{\circ} \mathrm{E}$ and to the north of $38^{\circ} \mathrm{N}$. Weather stations selected for the present study are homogeneously distributed (Figure 1). Therefore, a simple arithmetic mean is used in calculating the regional average. 


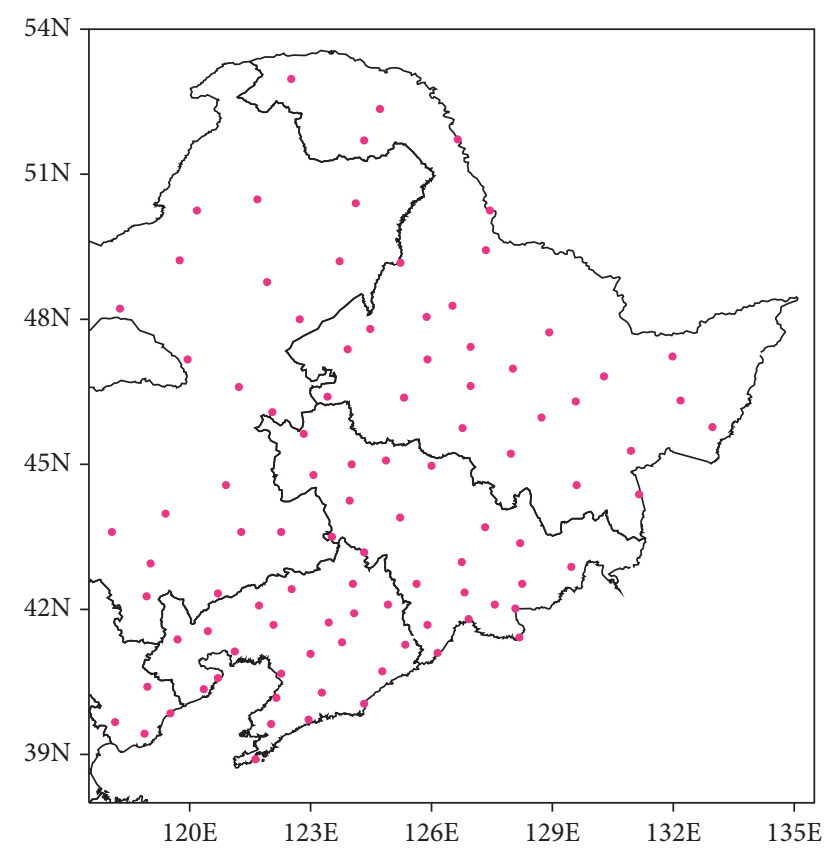

FIGURE 1: Distribution of 97 stations (dots) in Northeast China.

2.2. Method. Conventional statistical methods including composite analysis and $t$-test are used in the present study. Some dynamic diagnoses are also employed.

The horizontal component of wave activity flux $[31,32]$ (T-N flux) is employed to diagnose the disturbance energy propagation. Note that the T-N flux is independent of the wave phase under the WKB approximation and is consistent with the local group velocity direction of the stationary Rossby wave. The remainder of the T-N flux relative to the phase velocity is $\mathbf{W}_{\mathrm{r}}$, which can be expressed as

$$
\mathbf{W}_{\mathrm{r}}=\frac{p}{2000|\mathbf{U}|}\left(\begin{array}{c}
U\left(v^{\prime 2}-\psi^{\prime} v_{x}^{\prime}\right)+V\left(-u^{\prime} v^{\prime}+\psi^{\prime} u_{x}^{\prime}\right) \\
U\left(-u^{\prime} v^{\prime}+\psi^{\prime} u_{x}^{\prime}\right)+V\left(u^{\prime 2}+\psi^{\prime} u_{y}^{\prime}\right)
\end{array}\right)
$$

where $\psi^{\prime}$ is the disturbance of quasi-geostrophic streamfunction, $u^{\prime}$ and $v^{\prime}$ are disturbance of quasi-geostrophic winds, $\mathbf{U}=(U, V)$ is the basic flow, and $p$ is the pressure. The basic flow in the above equation consists of zonal varying zonal and meridional winds, which is appropriate to describe meandering mid-high latitude background circulation.

Only the T-N flux at $200 \mathrm{hPa}$ is calculated in the present study. The climatological summer mean horizontal wind field is used as the basic flow field, and the disturbances refer to differences between horizontal winds on the days of extreme precipitation and the basic flow.

\section{The Regional Precipitation Extreme Events}

Usually, a daily precipitation extreme is identified for the individual station by selecting the events with precipitation amount larger than the critical value at 99th percentile in the daily series of precipitation which is ranked in an increasing order [33, 34]. Based on this thinking, Guan et al. [7] and Ke and Guan [10] generalized this definition to a regional scale, and henceforth examined the daily extremes in a large area rather than at an individual station. In the present paper, we also investigate the daily precipitation extremes on a regional scale.

Daily precipitation collected at the selected 97 stations (Figure 1) in Northeast China in the summers of 1979 to 2014 (3312 days in total) is averaged to obtain regional averages for individual days, and the days with regionally averaged daily precipitation less than $1 \mathrm{~mm}$ are excluded. The remaining days are sorted in ascending order based on averaged daily precipitation. The value on the 99th percentile is taken as the threshold, and those days with averaged daily precipitation greater than this threshold $(22.2 \mathrm{~mm} / \mathrm{d})$ are taken as days of extreme precipitation event. According to the definition of extreme precipitation event, regionally averaged daily precipitation that falls above the 99th percentile of all precipitation events is defined as a regional (nonindividual site) extreme precipitation event $[7,10]$. A total of 26 extreme precipitation events are identified based on the above procedures and the date of each event is shown in Table 1 . It is seen from Table 1 that the occurrences of extreme precipitation events are more frequent during some years such as 1994, 2012, and 2013 whereas no events are found in some other years. These indicate that an interdecadal change has taken place in the past 35 years.

\section{Characteristics of Extreme Precipitation Events}

4.1. Spatial Distribution of Extreme Precipitation Events. In order to study the spatial distribution of regional extreme daily precipitation, precipitation averaged over the $26 \mathrm{ex}-$ treme events and its difference from the climatological summertime average are presented in Figure 2. The extreme precipitation shows a "low-high-low" pattern from northwest to southeast part of Northeast China. The maximum precipitation occurs in Liaoning Province, where the largest precipitation can be greater than $48 \mathrm{~mm} / \mathrm{d}$ (Figure 2(a)). The difference between the event-average of extreme precipitation and the mean climatology of summertime precipitation (Figure 2(b)) displays a "sandwiched" spatial pattern similar to that shown in Figure 2(a), and the largest difference of more than $44 \mathrm{~mm} / \mathrm{d}$ also appears in Liaoning Province.

4.2. Circulation Anomalies Conducive to Extreme Precipitation Events. Regional extreme daily precipitation events in Northeast China are directly associated with local circulation anomalies. The composite anomalies of rotational (streamlines) and divergent (vectors) wind components at $850 \mathrm{hPa}$ (a), $500 \mathrm{hPa}$ (b), and $200 \mathrm{hPa}$ (c) based on 26 extreme precipitation events are shown in Figure 3. 
TABLE 1: Dates of extreme precipitation events in the summers of 1979-2014.

\begin{tabular}{|c|c|}
\hline Year & Date \\
\hline 1979 & None \\
\hline 1980 & None \\
\hline 1981 & June 21, July 4 \\
\hline 1982 & None \\
\hline 1983 & None \\
\hline 1984 & August 10 \\
\hline 1985 & None \\
\hline 1986 & None \\
\hline 1987 & August 27 \\
\hline 1988 & None \\
\hline 1989 & July 18 \\
\hline 1990 & None \\
\hline 1991 & July 22, July 29 \\
\hline 1992 & None \\
\hline 1993 & None \\
\hline 1994 & July 13 , August 8 , August 16 \\
\hline 1995 & July 26 \\
\hline 1996 & None \\
\hline 1997 & August 21 \\
\hline 1998 & August 5 \\
\hline 1999 & None \\
\hline 2000 & None \\
\hline 2001 & None \\
\hline 2002 & None \\
\hline 2003 & August 6, August 22 \\
\hline 2004 & August 28 \\
\hline 2005 & None \\
\hline 2006 & None \\
\hline 2007 & None \\
\hline 2008 & August 1 \\
\hline 2009 & None \\
\hline 2010 & July 20, August 5 \\
\hline 2011 & None \\
\hline 2012 & July 22, July 29, August 4, August 29 \\
\hline 2013 & July 2, July 16 , August 16 \\
\hline 2014 & None \\
\hline
\end{tabular}

At $850 \mathrm{hPa}$ in the lower troposphere (Figure 3(a)), Northeast China is under the control of cyclonic circulation anomaly, which is favorable for the water vapor transport to Northeast China. The strong convergence occurs on the southeastern part of the cyclone anomaly. Such kind of strong low-level cyclonic circulation supports the occurrence of extreme precipitation.

In the middle troposphere $(500 \mathrm{hPa})$ (Figure 3(b)), the cyclonic circulation anomaly shifts northwestward compared with that at $850 \mathrm{hPa}$, while obvious anticyclonic circulation anomaly appears to the southeast of the cyclonic anomaly. From the South China Sea to Northeast China, the circulation still exhibits a cyclone-anticyclonecyclone wave train pattern though not that typical as described in some literatures such as [35-37].

At $200 \mathrm{hPa}$ in the upper troposphere (Figure 3(c)), large areas in the high-latitude region of Russia to the east of Lake Baikal are under the influence of anomalous cyclonic circulation, while the area from Northeast China to Japan is affected by anomalous anticyclonic circulation. Meanwhile, the western Pacific region to the east of Japan is under the influence of anomalous cyclonic circulation. Northeast China is located exactly under the divergence area in the upper troposphere that corresponds to the abnormal anticyclone. Comparing with the pattern at $850 \mathrm{hPa}$ (Figure 3(a)), it is clear that the circulation exhibits a significant baroclinic structure (Figure 3(a)).

Strong ascending motion is favorable for the occurrence of extreme precipitation. Meridional-vertical cross section of vertical circulation anomaly over $\left(117.5-137.5^{\circ} \mathrm{E}\right)$ is displayed in Figure 4, which shows two symmetrical meridional circulation cells to the south and north of Northeast China. The ascending branches of two meridional circulations overlap above Northeast China, leading to abnormally strong updrafts there. Maximum ascending motion appears within the layer of $300-600 \mathrm{hPa}$ near $45^{\circ} \mathrm{N}$, with the average vertical velocity equal to or larger than $12 \mathrm{~Pa} / \mathrm{s}$ in the central area of the updraft. This kind of largescale vertical motion provides dynamic condition necessary for the water vapor convergence and upward transport, which eventually leads to the formation of clouds and precipitation.

4.3. Water Vapor Transport during Extreme Precipitation Events. Extreme precipitation can only occur with sufficient water vapor supply. Composite anomalies of rotational and divergent components of vertically integrated water vapor flux for the 26 extreme events are presented in Figure 5, which shows that the spatial pattern of rotational component of water vapor flux anomaly is basically consistent with the rotational component of wind anomaly at $850 \mathrm{hPa}$. Northeast China is located in the strong convergence area between the southeastern part of the cyclonic circulation anomaly and the northwestern part of the anticyclonic circulation anomaly. The strong southwesterly between the two circulation anomalies continuously transport water vapor from the ocean to Northeast China, providing sufficient water vapor supply for extreme daily precipitation there. Abundant water vapor supply and strong ascending motion are the prerequisite for heavy precipitation. Note that the divergent component of water vapor flux indicates that the water vapor is also from the Inner Mongolia, Russia, the Sea of Japan, and the mid-high latitude region of northwestern Pacific, suggesting that extreme daily precipitation in Northeast China is actually related to convergence of water vapor transported from various regions.

\section{Wave Activities and SST Anomalies Conducive to Extreme Precipitation Events}

The direction of wave activity flux represents the energy propagation direction. Composite wave activity flux and its divergence at $200 \mathrm{hPa}$ based on 26 extreme daily precipitation events excluding the basic flow are displayed in Figure 6, which shows that the disturbance energy accumulates and converges in Northeast China. The wave energy accumulation in Northeast China is quite favorable for the 


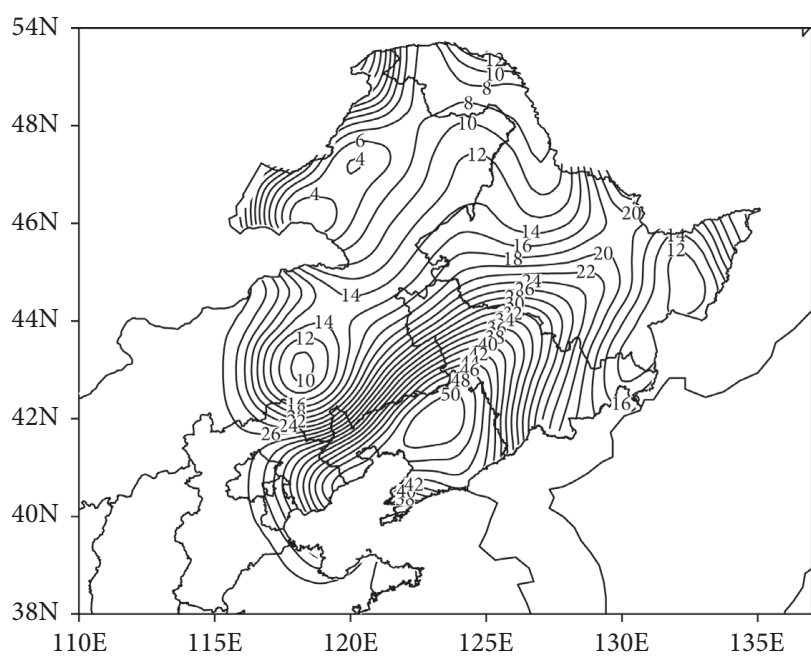

(a)

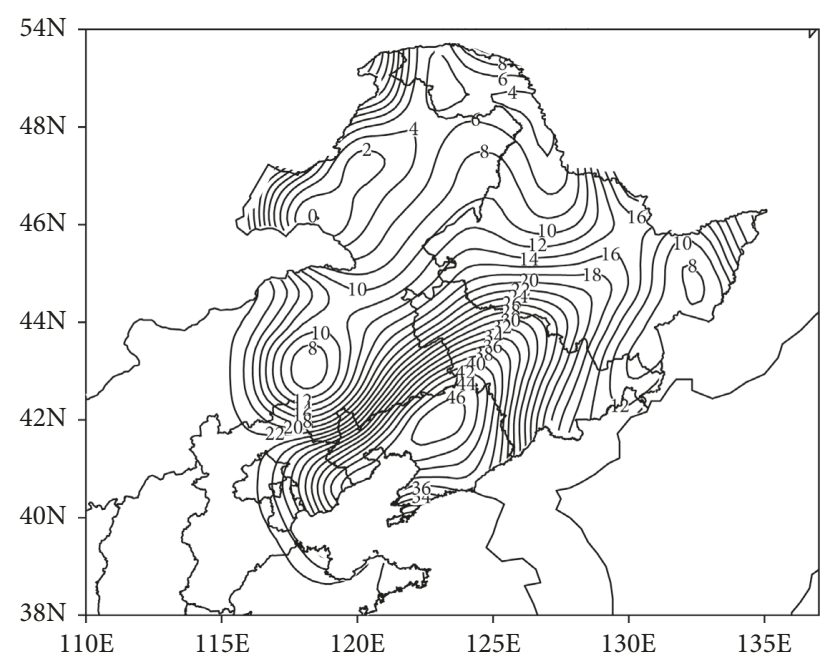

(b)

FIgURe 2: Distribution of precipitation amount $(\mathrm{mm} / \mathrm{d})$ averaged over the 26 extreme events (a) and that of the difference between the 26 case mean precipitation and the summertime mean climatology of the precipitation (b).

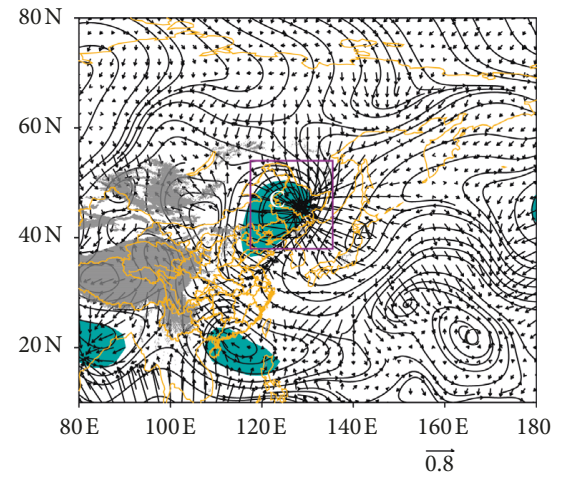

(a)

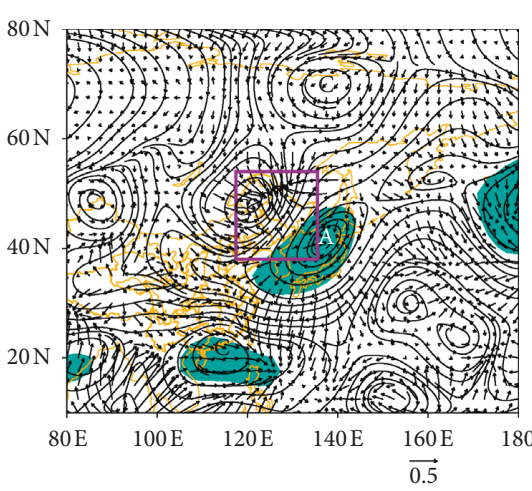

(b)

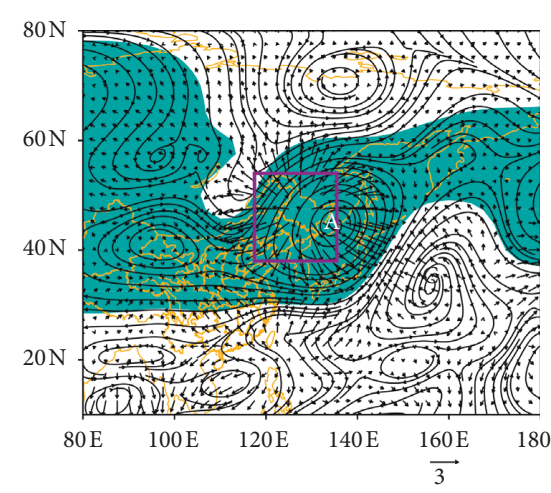

(c)

FIGURE 3: Composite anomalies of rotational (streamlines) and divergent (vectors) wind components (in $\mathrm{m} / \mathrm{s}$ ) at $850 \mathrm{hPa}$ (a), $500 \mathrm{hPa}$ (b), and $200 \mathrm{hPa}$ (c) based on 26 extreme precipitation events. Shaded areas indicate stream-functions at/above the $95 \%$ confidence level by using a $t$-test.

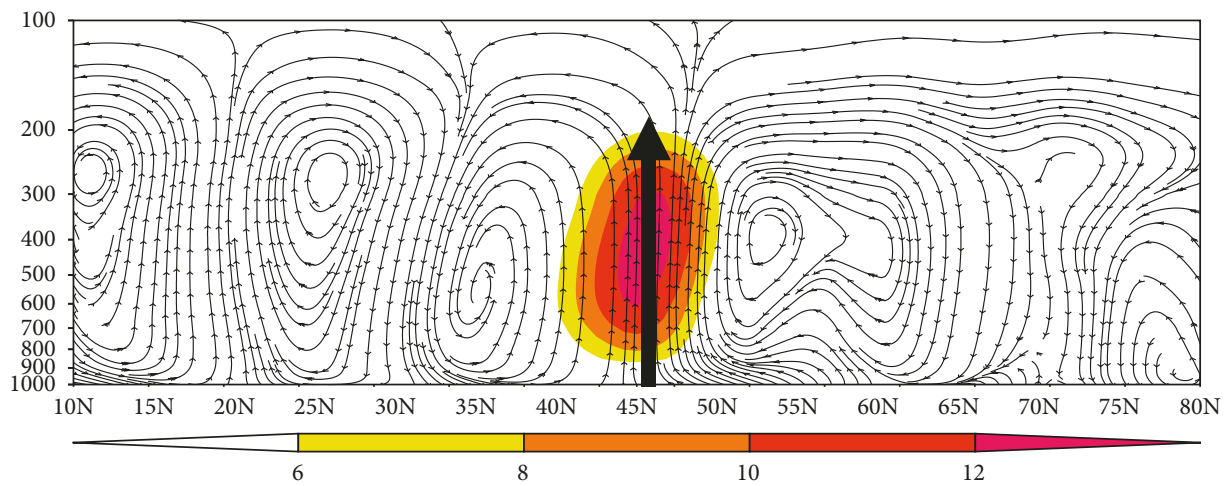

FIgURe 4: Meridional-vertical cross section of vertical circulation anomaly. Shaded area is for relatively larger vertical velocity $\omega$ (multiplied by 100$)$. 


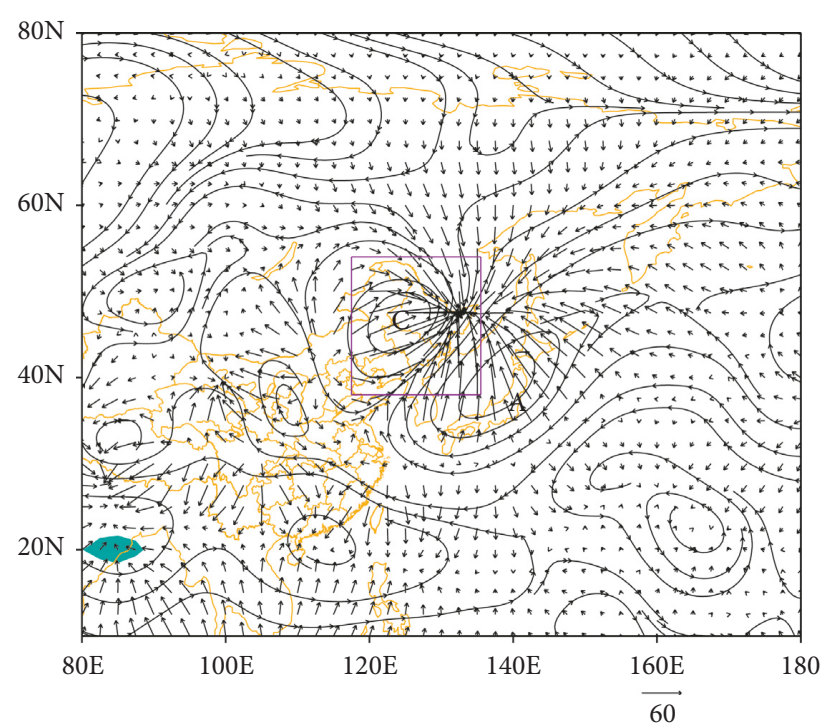

FIGURE 5: Composite anomalies of rotational and divergent (vectors, in $\mathrm{kg} / \mathrm{m} / \mathrm{s}$ ) components of vertically integrated water vapor flux based on 26 extreme events.

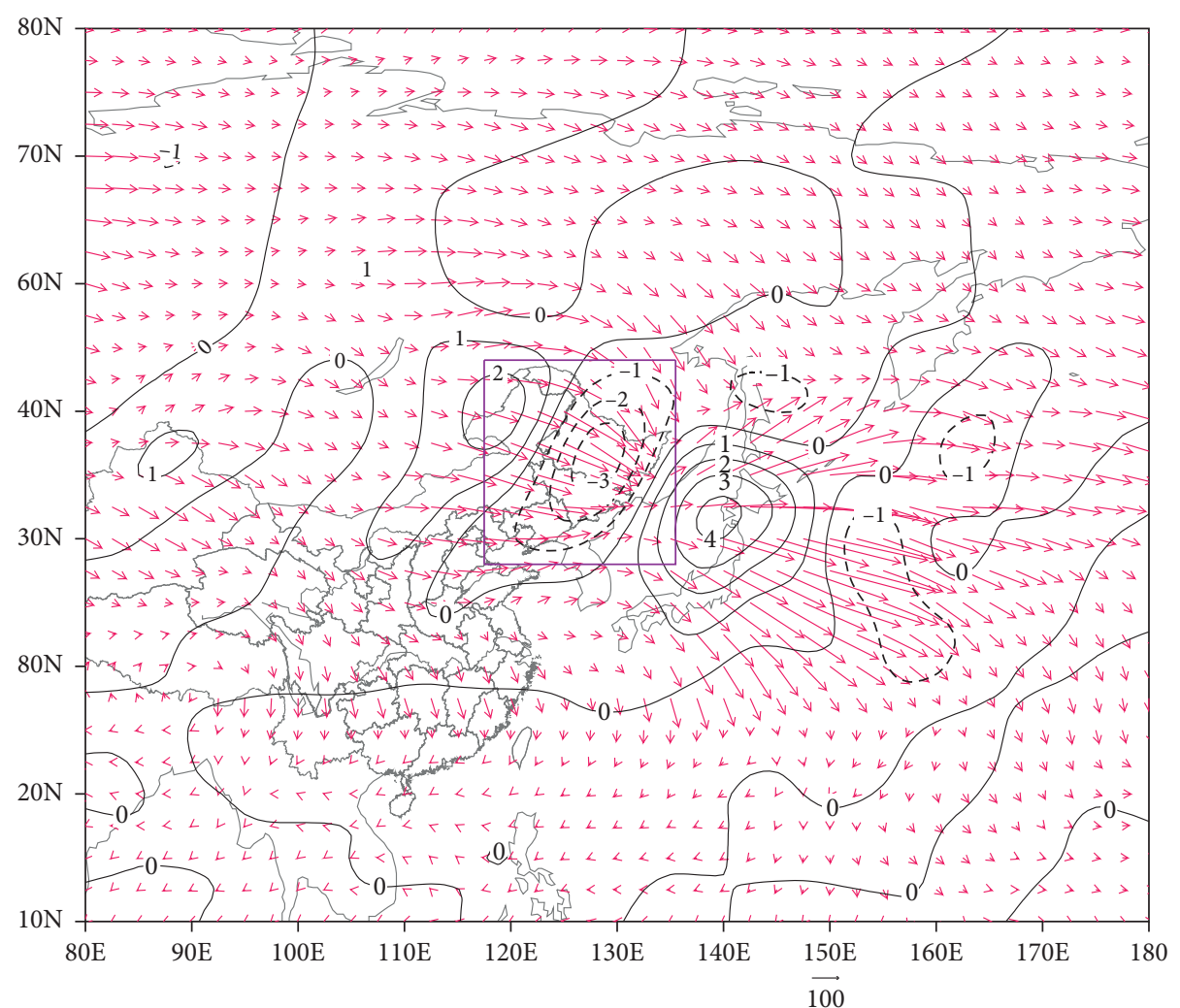

Figure 6: Composite wave activity flux (vectors, in $\mathrm{m}^{2} / \mathrm{s}^{2}$ ) and its divergence (contours, in $10^{-4} \mathrm{~m} / \mathrm{s}^{2}$ ) at 200 hPa based on 26 extreme daily precipitation events.

development and maintenance of anticyclonic perturbation at $200 \mathrm{hPa}$ above Northeast China and promotes the occurrence of extreme daily precipitation.

The occurrence of regional extreme daily precipitation in Northeast China is possibly related to SST anomalies. Composite difference between SST in the months with extreme precipitation events and climatological mean summer
SST is displayed in Figure 7, which shows the SST anomaly in the Pacific Ocean is roughly characterized by the feature of "positive-north and negative-south" with the equator as the boundary. Significant positive SST anomaly occurs over most areas of the Pacific to the north of $10^{\circ} \mathrm{N}$, with the maximum positive anomaly of greater than $2.4^{\circ} \mathrm{C}$ located near the Sea of Japan. 


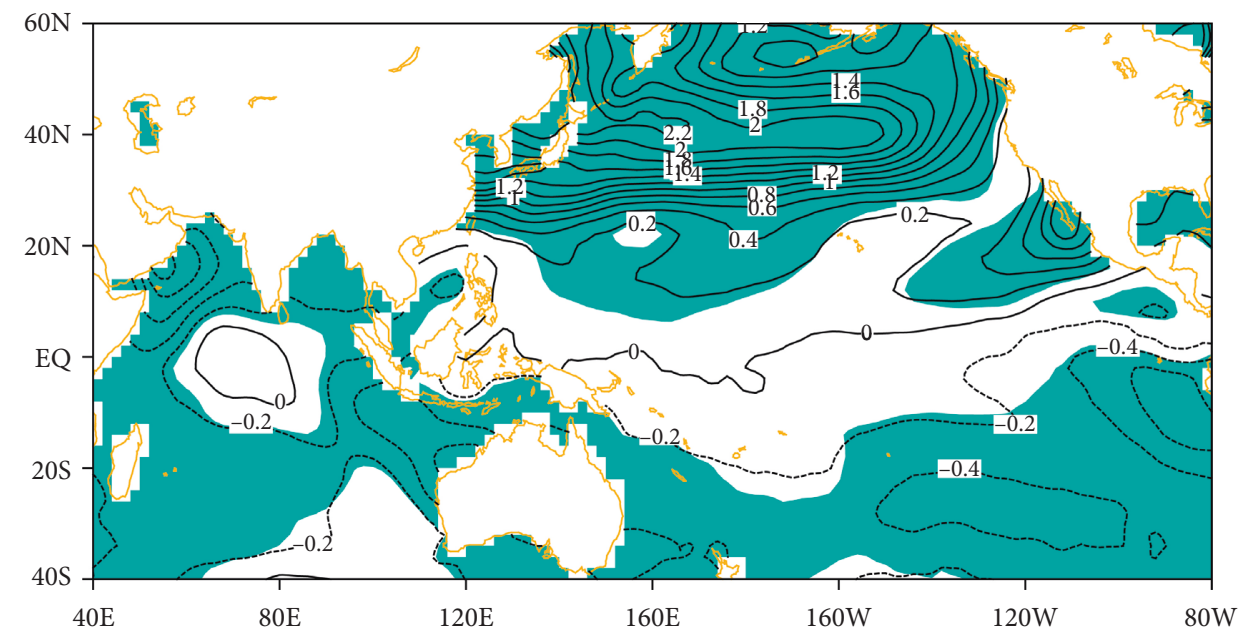

FIGURE 7: Composite difference between SST in the months with extreme precipitation events and climatological mean summer SST in ${ }^{\circ} \mathrm{C}$. Shaded areas indicate the $95 \%$ confidence level by $t$-test.

The linkage between SSTA pattern and precipitation extremes in Northeast China may be postulated. As seen in Figures 3(a) and 5, the airflow and water vapor converge from the region east of Japan archipelagos, where the SSTA is higher than normal. This means that the warmer anomalies of SST in mid-latitude North Pacific may facilitate the occurrences of extreme precipitation in Northeast China.

It should be noted that given the wind anomaly pattern over the North Pacific (Figure 3(a)), it appears that the positive anomalous SST may be a response of ocean to the anomalous anticyclonic circulation over the North Pacific. However, over the Sea of Japan, there exists the anomalous convergence at $850 \mathrm{hPa}$ (Figure 3(a)), suggesting that the higher than normal SSTA may play an active role in forcing the atmosphere to respond. Over other part of the North Pacific where the positive SSTAs are observed, the SSTA may play as a passive role in the ocean-atmosphere system by responding to the anomalous atmospheric circulation.

Note that the pattern with "positive-north and negativesouth" that is asymmetric about equator is quite different from the ENSO-SSTA pattern. In the previous studies, it is found that the ENSO has a good relation with the cold vortex in Northeast China [38], suggesting that during period of some cold vortexes, there occur some strong rainfall events. However, the SSTA pattern in Figure 7 does not look like the ENSO pattern, suggesting that the regionally mean daily precipitation extremes in northeast China may not closely relate to the ENSO.

\section{Summary}

Based on the above results, we conclude the present study as follows.

The precipitation on the 99th percentile is $22.2 \mathrm{~mm} / \mathrm{d}$, which is taken as the threshold for regional extreme daily precipitation in the summer in Northeast China. Regional extreme daily precipitation in the summer in Northeast China demonstrates significant interannual and interdecadal variations. In the 36 summers during 1979-2014, there are
26 extreme daily precipitation events in total. The spatial distribution of extreme precipitation shows a low-high-low feature from northwest to southeast, with the maximum value of up to $48 \mathrm{~mm} / \mathrm{d}$ occurring in Liaoning Province.

Circulation anomalies conducive to extreme daily precipitation based on the 26 extreme events indicate that Northeast China is located exactly below the divergence zone in the upper troposphere and within the strong convergence zone in the lower troposphere between the southeastern part of the cyclonic circulation anomaly and the northwestern part of the anticyclonic circulation anomaly. From the lower to upper troposphere, low-level convergence and upper-level divergence coexist above Northeast China, and the baroclinic structure in the vertical direction is favorable for the formation of extreme daily precipitation. Meanwhile, there exist two meridional circulation systems including the cyclone to the north and anticyclone to south of Northeast China. The ascending branches of the two circulations overlap over Northeast China, leading to abnormally strong ascending motion there. The vertically integrated water vapor flux anomalies of the 26 extreme events indicate that Northeast China is located in the strong convergence zone between the southeastern part of the cyclonic circulation anomaly and the northwestern part of the anticyclonic circulation anomaly. The water vapor supplies for extreme precipitation are not only from oceanic regions such as the Sea of Japan and mid-high latitudes of the northwestern Pacific but also from the inland regions including the Inner Mongolia and Russia.

The T-N fluxes of atmospheric wave activity and SST anomaly are favorable for the accumulation and convergence of disturbance energy in Northeast China. The wave energy accumulation in Northeast China is favorable for the development and maintenance of disturbance in Northeast China, which subsequently promotes the occurrence of extreme daily precipitation. Regional extreme daily precipitation in the summer in Northeast China is possibly related to SST anomaly. Corresponding to extreme precipitation events, SST 
anomaly in the Pacific Ocean is roughly characterized by the feature of "positive-north and negative- south" with the equator as the boundary. Significant positive SST anomaly occurs over most areas of the Pacific to the north of $10^{\circ} \mathrm{N}$, with the maximum positive anomaly of greater than $2.4^{\circ} \mathrm{C}$ located near the Sea of Japan.

Note that as the SSTA in Pacific does not clearly show the ENSO-like pattern, how this SSTA pattern with southnegative-north-positive SSTAs affects the rainfall extremes in Northeast China deserves further deep investigations in the future.

\section{Data Availability}

The data used in the present study are provided by National Meteorological Information Center of China Meteorological Administration, Information Service Center of Nanjing University of Information Science and Technology, and the NCEP/NCAR reanalysis product is provided by NOAA-CIRES Climate Diagnostics Center (http:// www.cdc.noaa.gov).

\section{Conflicts of Interest}

The authors declare that they have no conflicts of interest.

\section{Acknowledgments}

The data used in the present study are provided by National Meteorological Information Center of China Meteorological Administration, Information Service Center of Nanjing University of Information Science and Technology, and the NCEP/NCAR reanalysis product is provided by NOAACIRES Climate Diagnostics Center(http://www.cdc.noaa.gov). This study was supported by the China Meteorological Administration Special Public Welfare Research Fund (GYHY201406024), China Meteorological Administration Forecaster Special Fund (CMAYBY2015-015), and China Meteorological Administration Forecast Core Business Development Special Fund (CMAHX20160103).

\section{References}

[1] S. Zhao, Y. Deng, and R. X. Black, "A dynamical and statistical characterization of U.S. Extreme precipitation events and their associated large-scale meteorological patterns," Journal of Climate, vol. 30, no. 4, pp. 1307-1326, 2017.

[2] B. Z. Shen, Z. D. Lin, R. Y. Lu, and Y. Lian, "Circulation anomalies associated with interannual variation of early- and late-summer precipitation in Northeast China," Science China Earth Sciences, vol. 54, no. 7, pp. 1095-1104, 2011.

[3] P. Zhai, X. Zhang, H. Wan, and X. Pan, "Trends in total precipitation and frequency of daily precipitation extremes over China," Journal of Climate, vol. 18, no. 7, pp. 1096-1108, 2005.

[4] J. H. Yang, Z. H. Jiang, and P. X. Wang, "Temporal and spatial characteristic of extreme precipitation event in China," Climatic and Environmental Research, vol. 13, no. 1, pp. 75-83, 2008.

[5] Q. Dong, X. Chen, and T. Chen, "Characteristics and changes of extreme precipitation in the Yellow-Huaihe and Yangtze-
Huaihe Rivers Basins, China," Journal of Climate, vol. 24, no. 14, pp. 3781-3795, 2011.

[6] M. Gemmer, T. Fischer, T. Jiang, B. Su, and L. L. Liu, "Trends in precipitation extremes in the Zhujiang River Basin, South China," Journal of Climate, vol. 24, no. 3, pp. 750-761, 2011.

[7] Z. Y. Guan, J. Han, and M. G. Li, "Circulation patterns of regional mean daily -precipitation extremes over the middle and lower reaches of the Yangtze River during the boreal summer," Climate Research, vol. 50, no. 2, pp. 171-185, 2011.

[8] L. P. Li, G. Y. Xu, L. P. Cheng et al., "Characteristics of extreme precipitation and its variation trend in the post-flood of South China," Transactions of Atmospheric Sciences, vol. 35, no. 5, pp. 570-577, 2012, in Chinese.

[9] M. G. Li, Z. Y. Guan, J. Han et al., "Interdecadal changes of summertime precipitation extremes in East China in recent five decades," Transactions of Atmospheric Sciences, vol. 35, no. 5, pp. 591-602, 2012, in Chinese.

[10] D. Ke and Z. Y. Guan, "Variations in regional mean daily precipitation extremes and related circulation anomalies over central China during boreal summer," Journal of Meteorological Research, vol. 28, no. 4, pp. 524-539, 2014.

[11] Y. Y. Long, G. Z. Fan, D. Lian et al., "A study on the characteristics of summertime extreme precipitation events over China in recent 54 years," Climatic and Environmental Research, vol. 21, no. 4, pp. 429-438, 2016, in Chinese.

[12] J. Sun and F. Zhang, "Daily extreme precipitation and trends over China," Science China Earth Sciences, vol. 60, no. 12, pp. 2190-2203, 2017.

[13] Y. Deng, W. Jiang, B. He, Z. Chen, and K. Jia, "Change in intensity and frequency of extreme precipitation and its possible teleconnection with large-scale climate index over the China from 1960 to 2015," Journal of Geophysical Research: Atmospheres, vol. 123, no. 4, pp. 2068-2081, 2018.

[14] W. B. Wu, Q. L. You, D. Wang et al., "Characteristics of extreme precipitation and associated anomalous circulations over eastern China during boreal summer," Climatic and Environmental Research, vol. 23, no. 1, pp. 47-58, 2018, in Chinese.

[15] S. Y. Yang, F. H. Sun, and J. Z. Ma, "Evolvement of precipitation extremes in Northeast China on the background of climate warming," Scientia Geographica Sinica, vol. 28, no. 2, pp. 224-228, 2008.

[16] H. S. Chen, Q. D. Fan, and X. H. Zhang, "Seasonal differences of variation characteristics of extreme precipitation events over China in the last 50 years," Transactions of Atmospheric Sciences, vol. 32, no. 6, pp. 744-751, 2009.

[17] P. Qin and Z. Xie, "Precipitation extremes in the dry and wet regions of China and their connections with the sea surface temperature in the eastern tropical Pacific Ocean," Journal of Geophysical Research: Atmospheres, vol. 122, no. 12, pp. 6273-6283, 2017.

[18] B. Zhou, P. Zhai, and R. Niu, "Comparative assessment of two objective forecast models for cases of persistent extreme precipitation events in the Yangtze-Huai River valley in summer 2016," Weather and Forecasting, vol. 33, no. 1, pp. 221-238, 2018.

[19] W. Li, Z. H. Jiang, J. J. Xu, and L. Li, "Extreme precipitation indices over China in CMIP5 models. Part II: probabilistic projection," Journal of Climate, vol. 29, no. 24, pp. 8989-9004, 2016.

[20] J. Wu, B. T. Zhou, and Y. Xu, "Response of precipitation and its extremes over China to warming: CMIP5 simulation and projection," Chinese Journal of Geophysics, vol. 58, no. 9, pp. 3048-3060, 2015, in Chinese. 
[21] Y. Chen and P. Zhai, "Precursor circulation features for persistent extreme precipitation in central-eastern China," Weather and Forecasting, vol. 29, no. 2, pp. 226-240, 2014.

[22] N. Liang, J. Liu, and B. Wang, "How does the South Asian high influence extreme precipitation over eastern China?," Journal of Geophysical Research: Atmospheres, vol. 122, no. 8, pp. 4281-4298, 2017.

[23] Q. Zhang, Y. Zheng, V. P. Singh, M. Luo, and Z. Xie, "Summer extreme precipitation in eastern China: mechanisms and impacts," Journal of Geophysical Research: Atmospheres, vol. 122, no. 5, pp. 2766-2778, 2017.

[24] Q. Zhang, X. H. Gu, J. F. Li, P. Shi, and V. P. Singh, "The impact of tropical cyclones on extreme precipitation over coastal and inland areas of China and its association to ENSO," Journal of Climate, vol. 13, no. 5, pp. 1865-1880, 2018.

[25] B. Bi, X. Zhang, and K. Dai, "Characteristics of 2016 severe convective weather and extreme rainfalls under the background of super El Niño," Chinese Science Bulletin, vol. 62, no. 9, pp. 928-937, 2017.

[26] X. Sun, G. Jiang, X. Ren et al., "Role of intraseasonal oscillation in the persistent extreme precipitation over theYangtze River Basin during June 1998," Journal of Geophysical Research: Atmospheres, vol. 121, no. 18, pp. 10,453-10,469, 2016.

[27] Q. Wang, S. L. Ling, and J. J. Fu, "The impacts of ssta in Kuroshio and its extension on precipitation in Northeast China under the background of the two different ENSO case," Journal of Tropical Meteorology, vol. 32, no. 1, pp. 73-84, 2016.

[28] G. Liu, G. Feng, Y. L. Qin et al., “"Cumulative effect” of cold vortex precipitation in Northeast China in early summer," Chinese Journal of Atmospheric Sciences, vol. 41, no. 1, pp. 202-212, 2017.

[29] H. S. Chen, J. Q. Yang, W. X. Zhang et al., "Possible linkages among early summer precipitation in Northeast China, coldvortex and spring land surface thermal anomaly over West Asia," Journal of Marine Meteorology, vol. 38, no. 1, pp. 10-16, 2018.

[30] E. Kalnay, M. Kanamitsu, R. Kistler et al., "The NCEP/NCAR 40-year reanalysis project," Bulletin of the American Meteorological Society, vol. 77, no. 3, pp. 437-471, 1996.

[31] K. Takaya and H. Nakamura, "A formulation of a wave-activity flux for stationary Rossby waves on a zonally varying basic flow," Geophysical Research Letters, vol. 24, no. 23, pp. 2985-2988, 1997.

[32] K. Takaya and H. Nakamura, "A formulation of a phaseIndependent wave-activity flux for stationary and migratory quasigeostrophic eddies on a zonally varying basic flow," Journal of the Atmospheric Sciences, vol. 58, no. 6, pp. 608$627,2001$.

[33] K. E. Kunkel, K. Andsager, and D. R. Easterling, "Long-term trends in extreme precipitation events over the conterminous United States and Canada," Journal of Climate, vol. 12, no. 8, pp. 2515-2527, 1999.

[34] R. Yamamoto and Y. Sakurai, "Long-term intensification of extremely heavy rainfall intensity in recent 100 years," World Resource Review, vol. 11, no. 2, pp. 271-281, 1999.

[35] T. Nitta, "Convective activities in the tropical western Pacific and their impact on the Northern Hemisphere summer circulation," Journal of the Meteorological Society of Japan. Ser. II, vol. 65, no. 3, pp. 373-390, 1987.

[36] R. H. Huang, "The east Asia/Pacific pattern teleconnection of summer circulation and climate anomaly in east Asia," Acta Meteorologica Sinica, vol. 6, no. 1, pp. 25-37, 1992.
[37] Z. Y. Guan and T. Yamagata, "The unusual summer of 1994 in East Asia:IOD teleconnections," Geophysical Research Letters, vol. 30, no. 10, pp. 1544-1547, 2003.

[38] Y. Lian, B. Shen, S. Li, G. Liu, and X. Yang, "Mechanisms for the formation of Northeast China cold vortex and its activities and impacts: an overview," Journal of Meteorological Research, vol. 30, no. 6, pp. 881-896, 2016. 

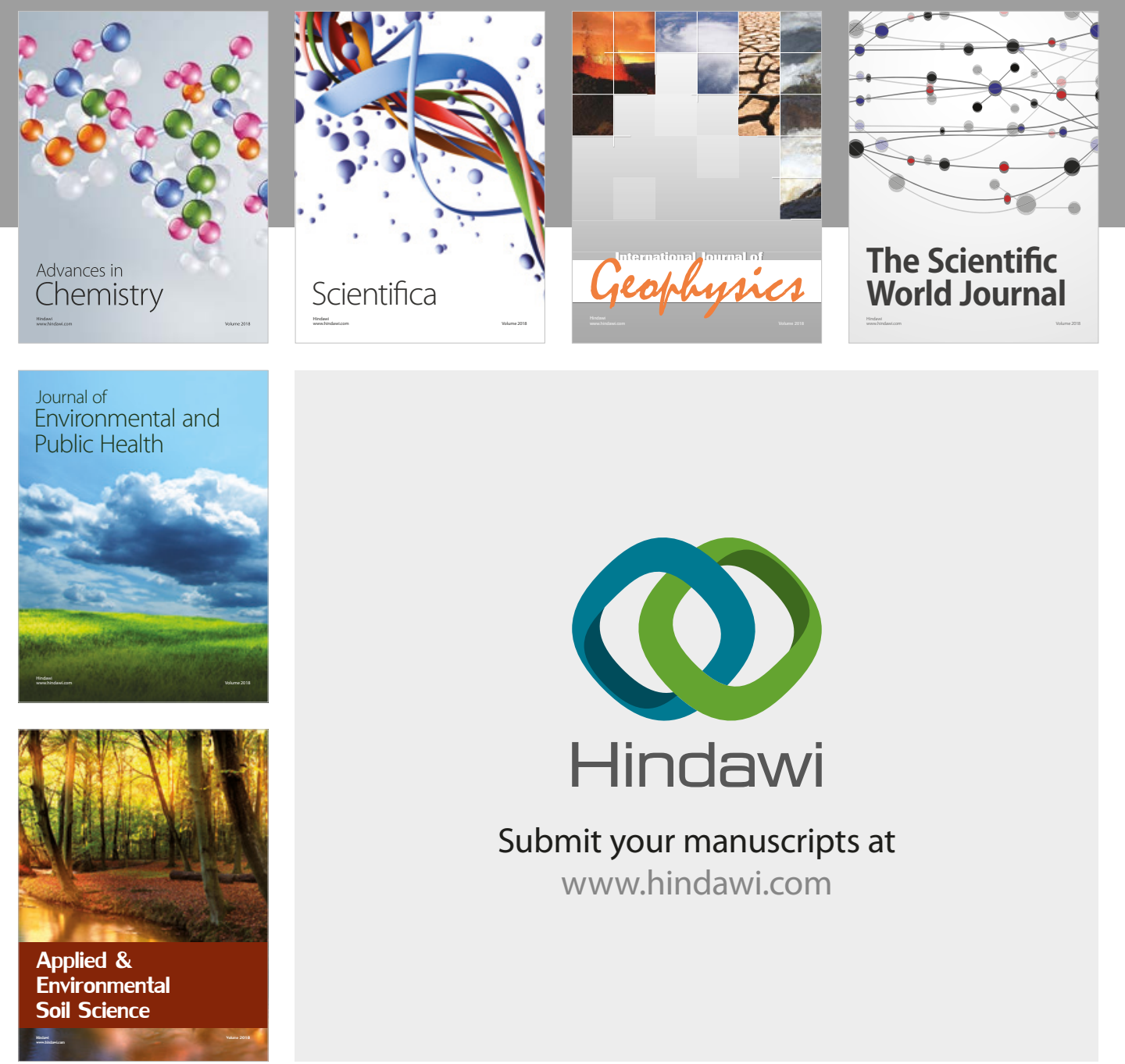

The Scientific

\section{World Journal}
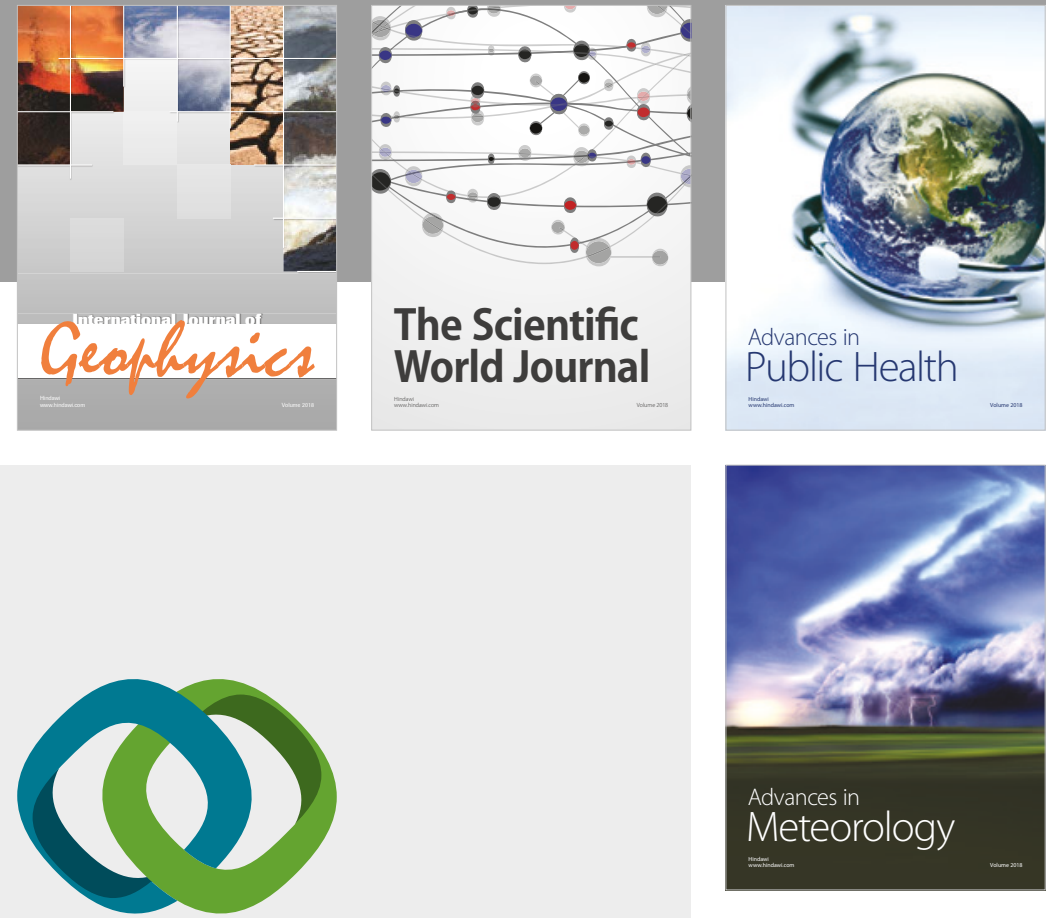

Advan

Public Health

\section{Hindawi}

Submit your manuscripts at

www.hindawi.com
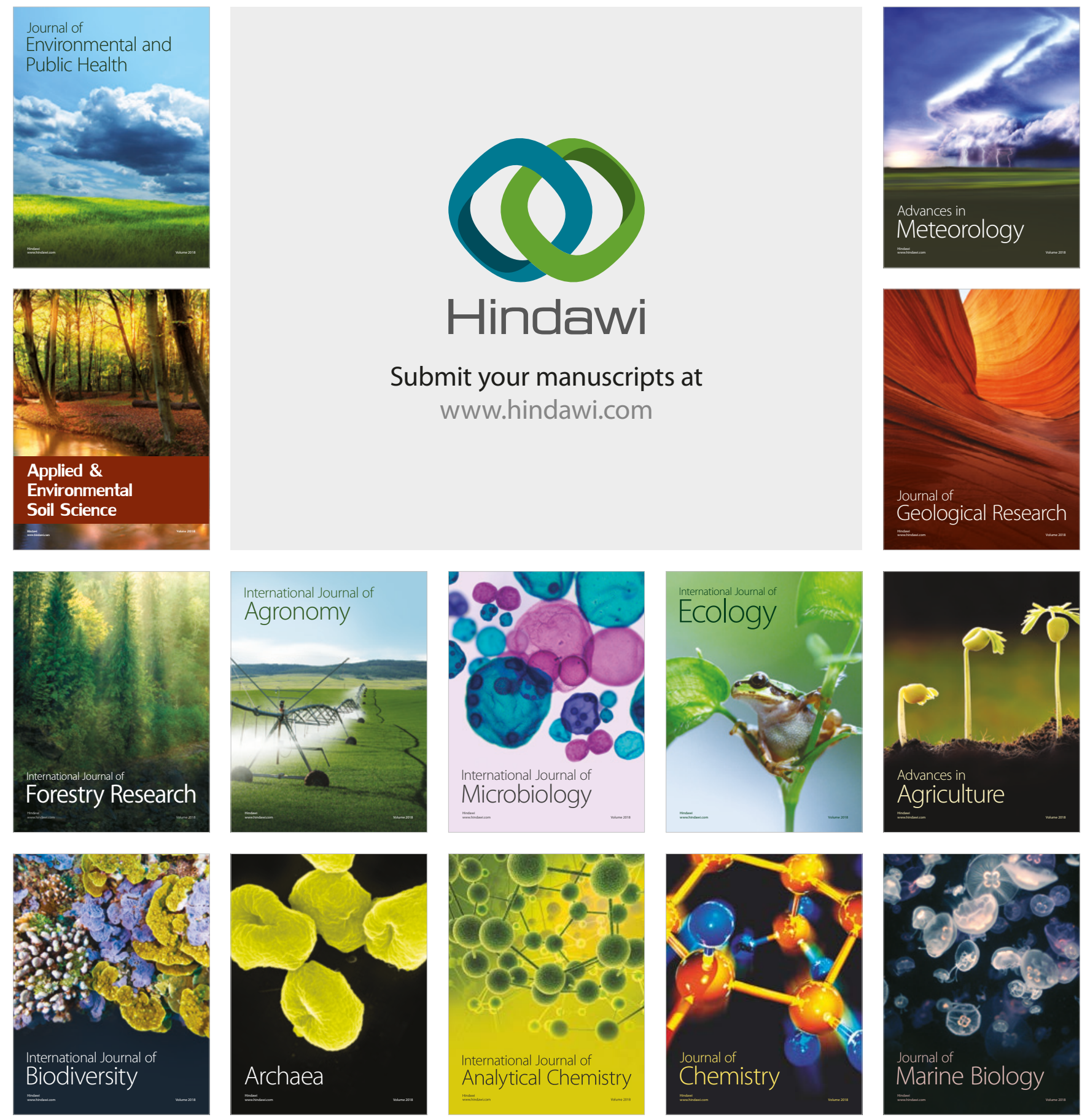